\title{
TIMI Coronary Thrombus Grade 5
}

National Cancer Institute

\section{Source}

National Cancer Institute. TIMI Coronary Thrombus Grade 5. NCI Thesaurus. Code C119587.

Total vessel occlusion. (Gibson, C. M., de Lemos, J. A., Murphy, S. A., Marble, S. J., McCabe, C. H., Cannon, C. P., Antman, E. M., Braunwald, E. Combination therapy with abciximab reduces ang iographically evident thrombus in acute myocardial infarction: a TIMI 14 substudy Circulation. 2001;103(21):2550-4.) 